\title{
The Temporal Development of Clinical Research in Emerging Countries
}

\author{
Vanessa Strüver $^{1, *}$, Firas Fneish ${ }^{2}$, Rainer Muche ${ }^{3}$, Gerhard Fortwengel $^{1}$ \\ ${ }^{1}$ Faculty III - Media Information and Design, Hochschule Hannover, Hannover, Germany \\ ${ }^{2}$ Institute for Cell Biology and Biophysics, Leibniz University Hannover, Hannover, Germany \\ ${ }^{3}$ Institute for Epidemiology and Medical Biometrics, Ulm University, Ulm, Germany

\section{Email address:} \\ vanessa.struever@hs-hannover.de (V. Strüver), firasfneish@gmail.com (F. Fneish), rainer.muche@uni-ulm.de (R. Muche), \\ gerhard.fortwengel@hs-hannover.de (G. Fortwengel) \\ ${ }^{*}$ Corresponding author
}

\section{To cite this article:}

Vanessa Strüver, Firas Fneish, Rainer Muche, Gerhard Fortwengel. The Temporal Development of Clinical Research in Emerging Countries. American Journal of Clinical and Experimental Medicine. Vol. 9, No. 1, 2021, pp. 1-7. doi: 10.11648/j.ajcem.20210901.11

Received: December 8, 2020; Accepted: December 15, 2020; Published: January 12, 2021

\begin{abstract}
The globalization of clinical research business is leading to a shift of clinical trials from Western countries to socalled emerging markets. This article should present the temporal development of clinical research business on the African continent. Further information should be gathered on favorite disease categories in which clinical trials are conducted. Using the ClinicalTrials.gov registry, trend data on the development of clinical research in the 47 countries listed on the African continent country list published by the World Health Organization were collected for the period from 2000 to 2018. Additionally, health data for the two main disease categories Communicable Diseases and Non-Communicable Diseases are taken from the Atlas on African Health Statistics of the World Health Organization for each country to be utilized in comparative analyses. Twenty African countries showed a strong development in clinical research business with continuous clinical trials conducted since 2000, while 5 countries had a delayed beginning of development. A further 19 countries show only sporadic performance. A comparison of the data for the disease categories Communicable Diseases and NonCommunicable Diseases shows that in 38 countries the number of clinical trials in the field of Communicable Diseases has decreased over the course of the reporting period, while clinical trials in the category of Non-Communicable Diseases are increasingly being conducted. The expected globalization trend is not evident in all African emerging markets. Due to various factors, the countries show different levels of development in the clinical research business.
\end{abstract}

Keywords: Emerging Markets, Developing Countries, Clinical Trials, Communicable Diseases, Non-communicable Diseases

\section{Introduction}

"The globalization of clinical research is gaining momentum" [1]. This globalization trend has also led to a shift in the business of clinical research from formerly North America, Western Europe and Australia to so-called emerging markets such as Eastern Europe, Latin America or Africa [2]. It can therefore be assumed that a significant increase in clinical trials has taken place in emerging markets [3]. But are there any data that can confirm this assumption?

In order to identify possible trends, the article describes the temporal development of clinical research activities in emerging markets. The first part of the article covers the fundamental aspect of whether the number of clinical trials has changed in the course of the study period. The second part then examines the two main disease categories that were investigated in clinical trials during this period. This allows drawing conclusions on the strength of globalization in emerging markets and thus on the balance of health care in these countries, as well as assessing the impact of clinical research on the burden of disease.

Since Africa is a continent with a high number of emerging markets, it was chosen as basis for data collection. All 47 countries of the World Health Organization's list of countries belonging to the African region were included in the data 
collection [4]. The ClinicalTrials.gov study registry was used to collect data on the number of clinical trials between 2000 and 2018. Beside country-specific study frequencies, study numbers for the main disease categories were also analyzed.

\section{Methods}

\subsection{A Search of Clinical Trial Databases}

To obtain a comprehensive overview of the temporal development in the clinical research business on the African continent, a corresponding database query was done in the ClinicalTrials.gov registry of the U.S. National Library of Medicine at the National Institutes of Health [5]. To complete the data set, this database query was also done in the local Pan African Clinical Trials Registry [6]. However, this registry contains only a small number of clinical trials, so no meaningful results were obtained and thus it was not included in the final analysis.

\subsection{Health Statistics of the World Health Organization}

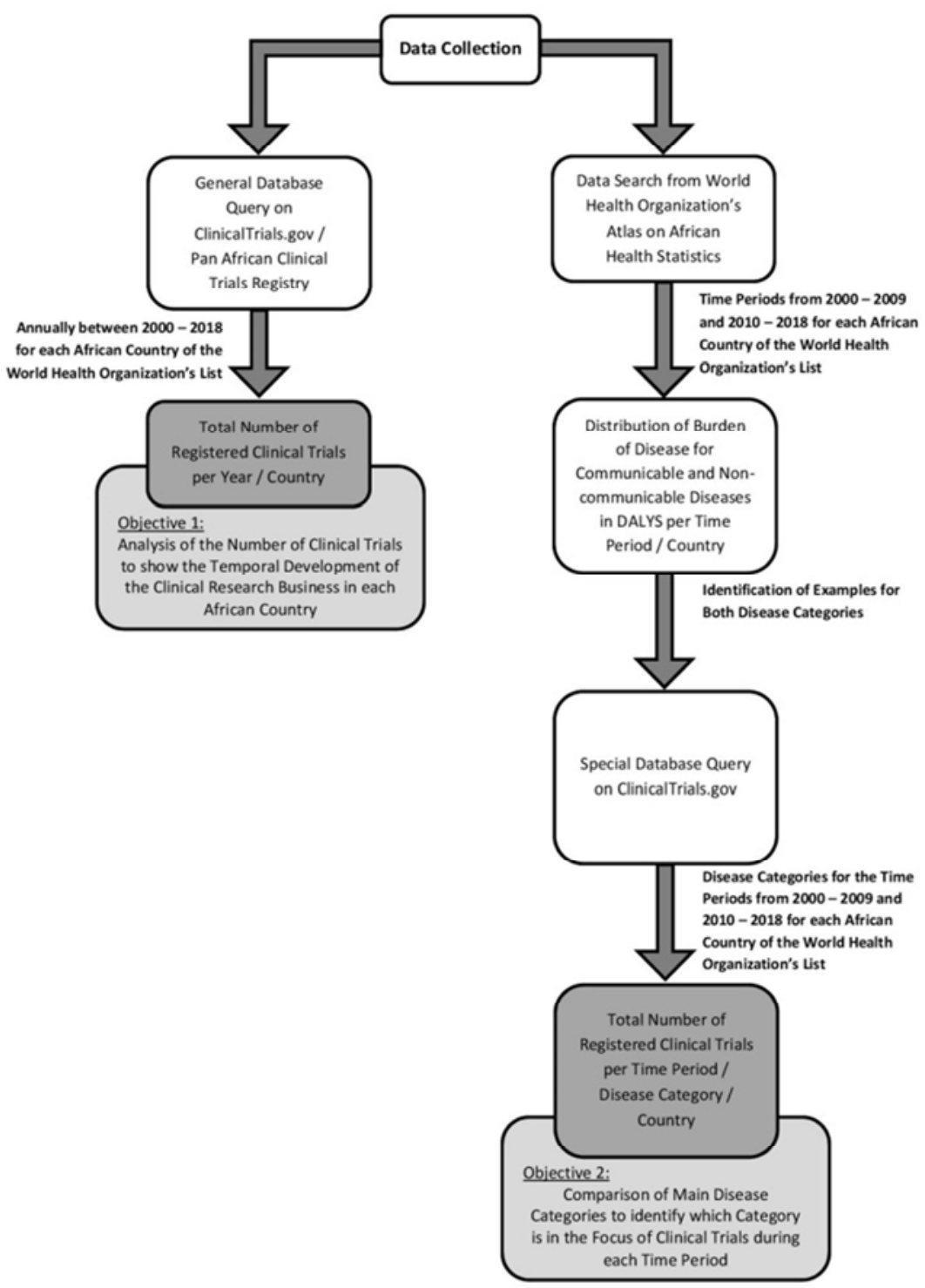

Figure 1. Methodology for data collection.

In determining whether the number of clinical trials conducted is influenced by various variables, a subanalysis was conducted in nine randomly selected countries of the African region to examine population size, gross national income and the development status of the health care system. The statistical data published by the World Health Organization for the respective country served as the data basis for this analysis [4]. The results of the subanalysis have shown that the researched factors do not allow any conclusions to be made regarding a possible influence on the number of clinical trials, since clinical trials are carried out to varying degrees independently of a large or small population, a high or low gross national income and a good or only rudimentarily developed health system. Thus, these factors 
receive no further consideration in the main analysis.

In a second step, health data from the Atlas of Health Statistics 2011 [7] as well as the Atlas of African Health Statistics 2016 [8], both published by the World Health Organization, were collected. Figure 1 gives an overview of the data collection methodology.

Data on the distribution of burden of diseases were collected in order to determine whether, in addition to a change in the business of clinical research, there is also a change in clinical trial indicational areas.

In order to portray a temporal development, the period from 2000 to 2018 was divided into two equal periods of time, so that the years 2000 to 2009 and the years 2010 to 2018 each gave a time span. For the period from 2000 to 2009, data from 2004 were used for the distribution of burden of diseases from the Atlas of Health Statistics 2011 of the Regional Office for Africa of the World Health Organization. From the Atlas of 2016 data from 2012 were extracted for the second investigational period. Out of the data listings, two data sets were tabulated containing data on occurrence of the main disease categories Communicable and Non-Communicable diseases per period. According to the Atlas of African Health Statistics, HIV / Aids, Lower Respiratory Infections, Malaria and Tuberculosis were selected as disease examples for the Communicable Diseases category. Stroke, Endocrine / Blood / Immune Disorders, Ischemic Heart Disease and Epilepsy served as disease examples for the category of Non-Communicable Diseases. The additional data on the injury category listed in the atlas were not further considered as they do not represent a specific disease category and therefore were irrelevant for answering the research question. Thus, the percentages taken for the distribution of communicable and non-communicable diseases were recalculated in order to reach a total of $100 \%$ with both figures.

\subsection{A search of Indication Areas in Clinical Trial Database}

The results of a second database query in ClinicalTrials.gov registry for Communicable and NonCommunicable diseases indication areas should demonstrate a potential correlation between disease categories and number of clinical trials. For each country, the number of clinical trials for the previously defined example diseases was determined for each time period and graphically presented in relative figures for comparative reasons.

\subsection{Data Analysis}

The collected data are then processed with the statistical software $\mathrm{R}$ version 3.6.1 to graphically represent the temporal development in the business of clinical research between the years 2000 and 2018 .

\section{Results}

\subsection{Search Result}

The general database query of the total number of clinical trials registered in the ClinicalTrials.gov study registry between 2000 and 2018 reflects a continuous development in clinical research throughout the period in the countries represented by Figure 2.

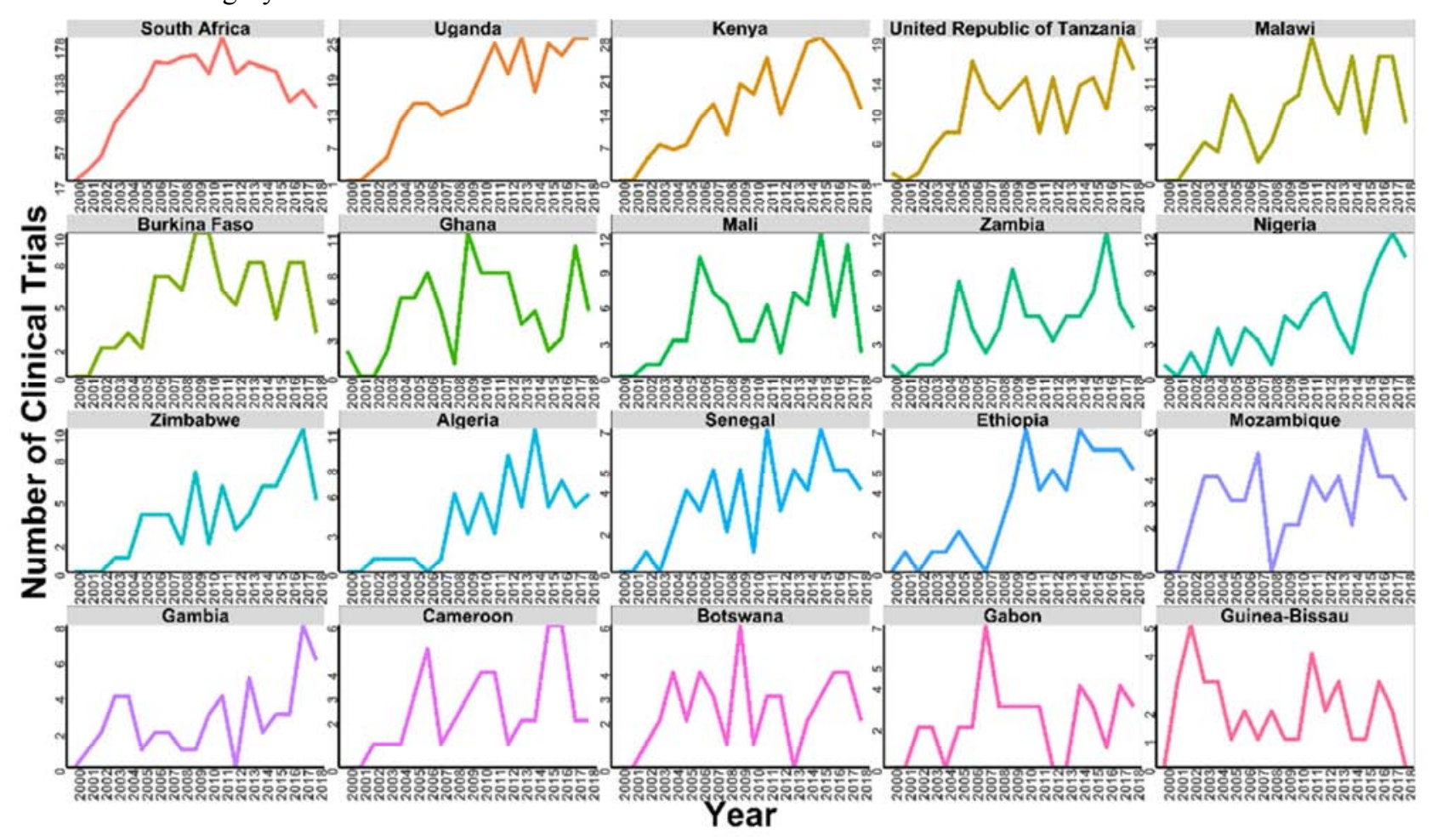

Figure 2. All countries demonstrating strong development in the conduct of clinical trials across the whole time period. 


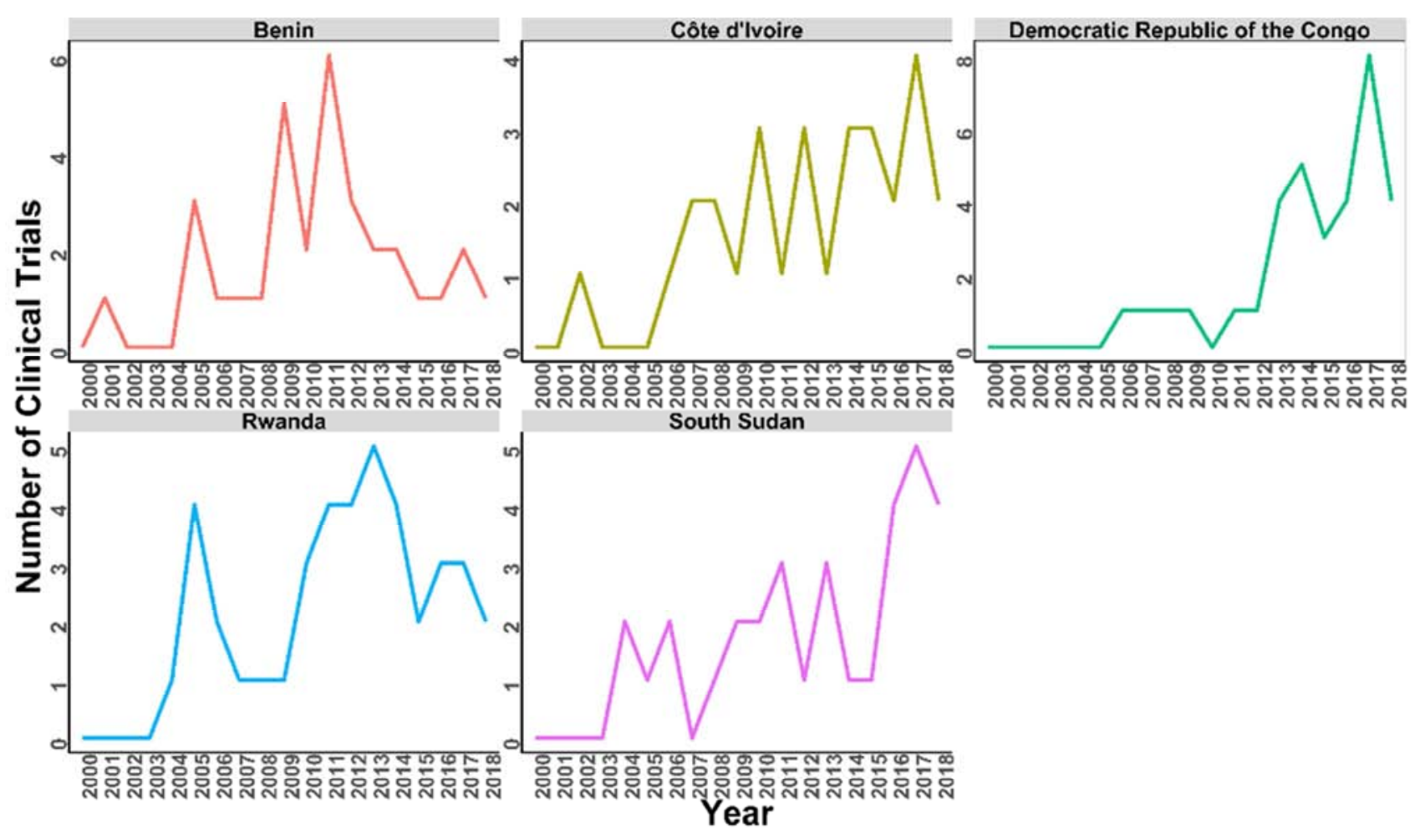

Figure 3. All countries that initiated their clinical trials later than 2003 and thus showed a delayed development in the conduct of clinical trials.

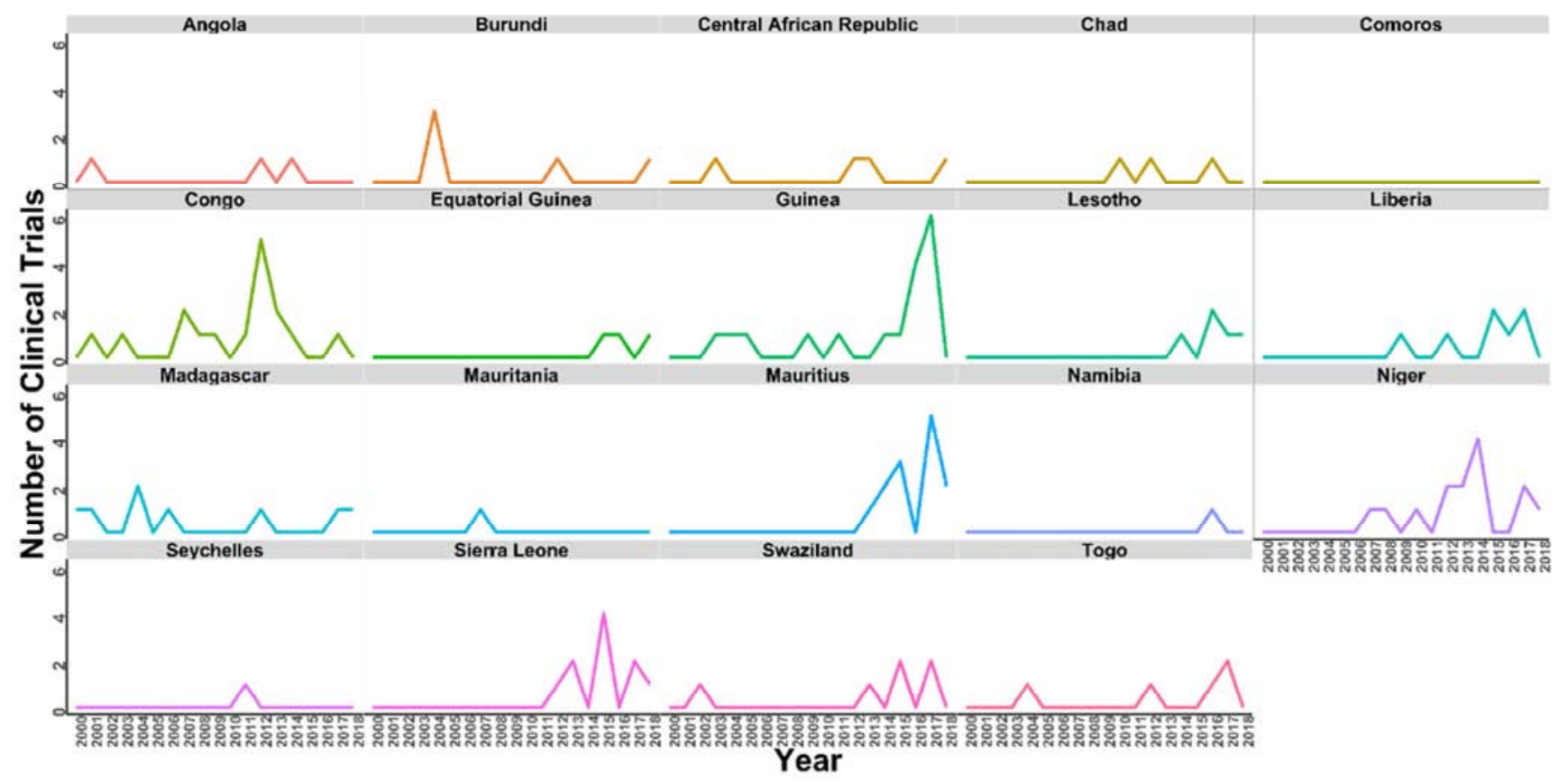

Figure 4. All countries showing sporadic clinical trials conduct.

In these countries, the number of clinical trials has increased in line with the expected upward trend in comparison between $2000-2003$ and 2018, although there are some up and down fluctuations in the years in between.

Figure 3 illustrates the countries whose total number of clinical trials indicates that development in the clinical research business has begun at a later time, since the start of clinical trials conduction is after 2003 and shows continued progress. In comparison, Figure 4 includes those countries which only have sporadic clinical trial conduction and thus do not record any temporal development in the business of clinical research.

The temporal development of clinical research business in Cape Verde, Eritrea and Sao Tome and Principe cannot be determined by the data collected as no trial data are available for these countries. 


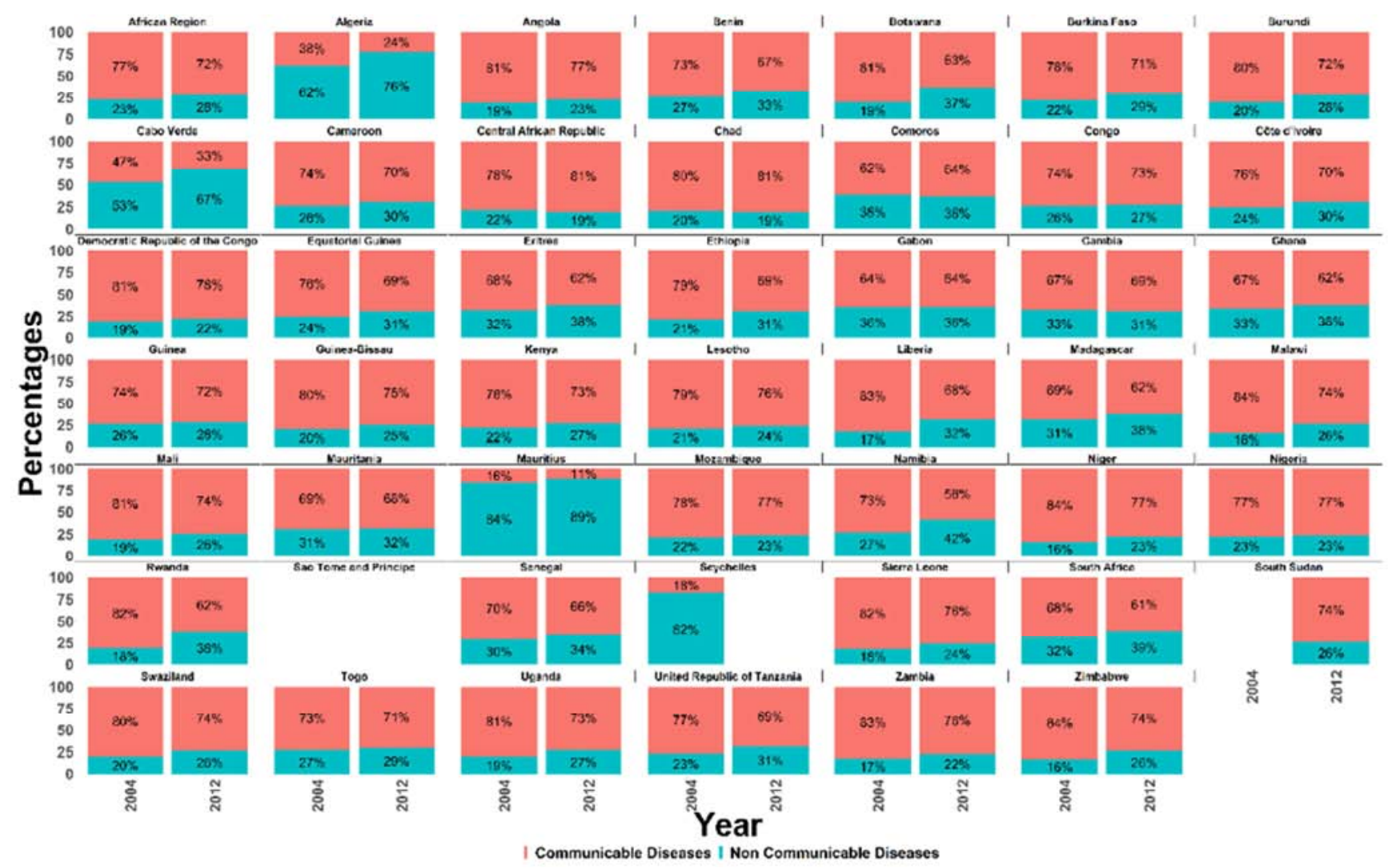

Figure 5. Comparison of the burden of disease distribution of the two main disease categories per country.

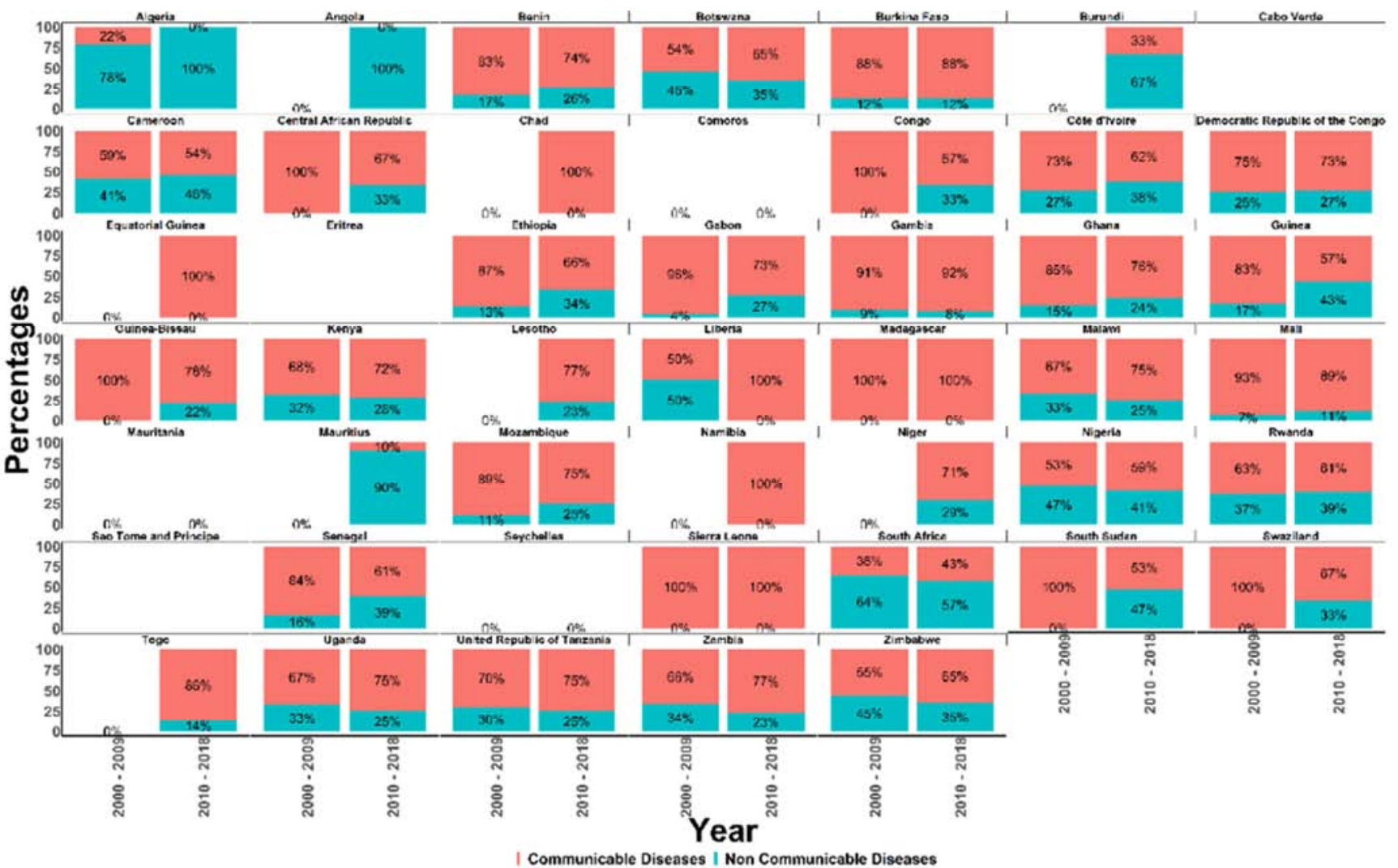

Figure 6. Comparison of the relative numbers (percentages) of clinical trials of the two main disease categories from 2000 - 2009 with those from 2010 - 2018. 


\subsection{Comparison of the Two Main Disease Categories Communicable Diseases and Non-communicable Diseases}

For each country, the burden of disease distribution of the two main disease categories Communicable Diseases and Non-Communicable Diseases is compared for each time period (Figure 5). It can be seen that in 38 of the 47 African countries there is a decrease in the frequency of the category Communicable Diseases compared to the years 2004 and 2012, while the category Non-Communicable Diseases increases in its frequency.

Figure 6 shows that in most African countries the general development in the business of clinical research can also be seen in the total number of clinical trials for the two disease categories. In 33 of the 47 African countries of the World Health Organization's list, the number of clinical trials in the Communicable Diseases category as well as in the category of Non-Communicable Diseases increased in both investigational periods (2000 - 2009 and 2010 - 2018). In 27 of the 47 countries, both the number of Communicable Diseases and Non-Communicable Diseases clinical trials increased.

Figure 6 also illustrates that in the period from 2000 to 2009 , the category of communicable diseases is much more in the focus of clinical trials than that of non-communicable diseases. In 30 out of 47 countries this category is found. Also, in the period 2010 - 2018, communicable diseases are more in the focus of clinical trials than non-communicable diseases in 36 of the 47 countries. Only Algeria and South Africa have a much higher number of clinical trials in the non-communicable disease category in both periods.

\section{Discussion}

Looking at various publications in the literature on globalization in clinical research, one thing becomes quite clear: in the past years there has been a shift in the conduct of clinical trials from developed countries such as the United States or Western Europe to emerging markets such as Eastern Europe, Latin America, Asia, the Middle East and Africa [2, 3, 9]. This trend can be confirmed for African countries as presented in Figures 2 and 3. Accordingly, 25 of the 47 African countries of the World Health Organization's country list of the African region support the higher frequency in the number of clinical trials. In contrast, there are also 19 countries which only record sporadic clinical trial conduction over the entire research period and further three countries without any documentation on clinical trials. These countries are obviously not experiencing this trend, which leads to the assumption that development depends on certain factors that are unlikely existing in these countries. As stated by Alemayehu et al, these factors include "ethical and regulatory issues, administrative issues, lack of finance, lack of infrastructure, poor data quality, and a lack of training" [10].
Although emerging countries have the greatest burden of disease in the world, some of them lack substantial research and development activities due to the factors mentioned. Even in these poorest regions "research-led solutions could bring the greatest impact to high rates of early mortality" [10]

Figure 5 represents that particularly communicable diseases, such as HIV/AIDS, tuberculosis, malaria or leprosy, are widespread in African countries and "still a major cause of mortality and morbidity" [11]. On the other side noncommunicable diseases, such as stroke, endocrine / blood / immune disorders, ischemic heart disease or epilepsy, also represent a burden for the population. In line with Alemayehu et al, clinical trials in emerging countries should mainly benefit the local population and therefore deal with the country-specific diseases, which predominantly belong to the category of communicable diseases [10]. Figure 6 supports this demand. It shows that between $2000-2009$ a total of 30 and between 2010 - 2018 as many as 36 of the 47 countries in the African region analyzed placed the focus of clinical trials on research into communicable diseases. The only exceptions are South Africa and Algeria. Both countries show a significantly higher interest in non-communicable diseases over the entire research period. One possible reason for this could be the strong development of tourism in the large cities of both countries. This fact has led to the adaptation of both countries to western habits and thus probably contributed to an increased awareness of the risk of infection with communicable diseases and their prevention on the one hand and to the spread of non-communicable diseases through a more modern, unhealthy lifestyle, e.g. through stimulants, on the other.

In total, however, the number of clinical trials in 27 countries has increased both in the area of communicable diseases and non-communicable diseases. This reflects the worldwide interest in conducting clinical trials. The alignment of the 10/90 gap could play an essential role here, so that in the future not only $10 \%$ of the worldwide funding will flow into health research "towards problems that affect $90 \%$ of the world's population", of which only a small percentage will go to "research and health problems indigenous to developing countries" [10]. In order to continue to advance clinical research in emerging countries, the formation of global health partnerships and collaborative research groups is a good opportunity to improve healthcare in Africa, by ensuring efficient and "important research in a resource constrained environment" [12]. Thus, future "headway can be made with the concerted efforts of governments, charities, foundations, product-development partnerships, academic institutions and pharmaceutical companies" [13].

\section{Limitations of the study}

The following limitations of the research work should be observed. Firstly, at the time of writing, no more recent data on the distribution of the burden of diseases by the World Health Organization have yet been available, although the Atlas of African Health Statistics 2018 has already been 
published. The atlas does not contain any data on the distribution of the burden of diseases, so that no conclusions could have been drawn about the current distribution of the burden of diseases.

Secondly, only the ClinicalTrials.gov study register, an internationally recognized database for the registration of clinical trials, was used to process the research question. Further research should therefore be carried out to determine whether local databases, apart from the Pan African Clinical Trials Registry, reflect a similar development in the business of clinical research or whether the changes are less evident at the local level.

\section{Conclusions}

Contrary to the actual expectations, the globalization trend, although confirmed in many countries, is not evident in all African emerging markets. Even those countries that have seen a substantial development in clinical research over the past twenty years show significant differences in the number of clinical trials conducted. These differences may be based on various barriers of trial implementation in these regions [10]. Based on "limited funds and a lack of trained staff to conduct their own research", it is not possible in every emerging country to carry out a mature clinical research, even if this would be necessary to treat diseases such as tuberculosis and malaria or to introduce appropriate prevention measures [14]. Doing so would be of enormous importance to translate research outcomes into practice and policy through education and involvement of local stakeholders to support dissemination of knowledge and integration into care [15]. All "developing countries urgently need research to help relieve the enormous burden of disease that they carry" and public as well as private sponsors in developed countries should finance research in developing countries to "help bridge this gap" [14]. Overall, the progress of clinical research activities thus already shows a noticeable trend toward globalization, although this has not yet taken effect in all African emerging markets and led to an improvement in health care. It can be assumed that globalization in these countries must continue to gain strength, with the purpose of using new research findings, new medicines and technologies to improve the existing burden of disease and the entire health care system.

\section{Conflict of Interest}

The authors declare that there is no conflict of interest.

\section{References}

[1] Ehni HJ, Wiesing U. Globalization in medical research. Chirurg 2018; 89 (3): 178-184.
[2] Jeong S, Sohn M, Kim JH, Ko M, Seo HW, Song YK et al. Current globalization of drug interventional clinical trials: characteristics and associated factors 2011-2013. Trials 2017; 18 (1): 288 .

[3] da Silva RE, Amorim Amato A, Guilhem DB, Carvalho Garbi Novaes MR. Globalization of clinical trials: ethical and regulatory implications. International Journal of Clinical Trials 2016; 3 (1): 1-8.

[4] World Health Organization - Regional Office for Africa (2017). WHO African Region Country Offices. https://www.afro.who.int/countries (accessed 30 July 2019).

[5] National Institutes of Health (NIH). U.S. National Library of Medicine ClinicalTrials.gov. https://clinicaltrials.gov/ (accessed 02 August 2019).

[6] Pan African Clinical Trials Registry (PACTR). Pan African Clinical Trials Registry. https://pactr.samrc.ac.za/ (accessed 05 August 2019).

[7] African Health Observatory. World Health Organization Regional Office for Africa (2011). Health Situation Analysis in the African Region - Atlas of Health Statistics, 2011. http://www.aho.afro.who.int/en/atlas/atlas-african-healthstatistics-2011 (accessed 22 July 2019).

[8] African Health Observatory. World Health Organization Regional Office for Africa (2016). Atlas of African Health Statistics 2016 - Health situation analysis of the African Region. http://www.aho.afro.who.int/en/atlas/atlas-africanhealth-statistics-2016-health-situation-analysis-of-the-africanregion (accessed 22 July 2019).

[9] Limaye D, Langer JM, Rühling T, Fortwengel G. A critical appraisal of clinical trials conducted and subsequent drug approvals in India and South Africa. BMJ Open 2015; 5: e007304.

[10] Alemayehu C, Mitchell G, Nikles J. Barriers for conducting clinical trials in developing countries - a systematic review. International Journal for Equity in Health 2018; 17: 37.

[11] De Rycker M, Horn D, Aldridge B, Amewu RK, Barry CE, Buckner FS et al. Setting Our Sights on Infectious Diseases. ACS Infectious Diseases 2020; 6: 3-13.

[12] Conradie A, Duys R, Forget P, Biccard BM. Barriers to clinical research in Africa: a quantitative and qualitative survey of clinical researchers in 27 African countries. British Journal of Anaesthesia 2018; 121 (4): 813-821.

[13] De Rycker M, Baragaña B, Duce SL, Gilbert IH. Challenges and recent progress in drug discovery for tropical diseases. Nature 2018; 559: 498-506.

[14] Nuffield Council on Bioethics. The ethics of research related to healthcare in developing countries - a guide to the Report. http://nuffieldbioethics.org/wpcontent/uploads/2014/07/HRRDC-short-Version-final.pdf (accessed 07 September 2019).

[15] Aluisio A, Waheed S, Cameron P, Hess J, Jacob ST, Kissoon $\mathrm{N}$ et al. Clinical emergency care research in low-income and middle-income countries: opportunities and challenges. BMJ Global Health 2019; 4: e001289. 